\title{
MODELLING THE ULTRASONIC DIAGNOSIS OF ADHESIVE BONDS
}

In the paper, the conception of modelling the diagnosis of adhesive bonds by ultrasonic method is presented. Two complementary models of bonds diagnosis are presented and discussed. The models proposed reduce an evaluation of coating adherence to assessment of condition of bond between coating and substrate. The models are connected with period of production of adhesive bonds (so called control diagnostic) and with the operational or maintenance period of its existence (operational diagnostic).

\section{Introduction}

As a results of own investigation of adhesive bonds between coatings and substrate a number of relationships between mechanical and ultrasonic parameters of strength of those bonds [1-3] were received. Some courses of gain of ultrasonic wave amplitude were also obtained with monitoring of the bonds by ultrasonic wave during their loading by bending moment on the special test stand [4]. These results give us a basis to the proposal of complementary diagnostic models of adhesive bonds between the coating and the substrate.

Those models formalize the coating adherence evaluation, reducing it to the assessment of the bond condition at various stages of its existence. Both the verification of the models and the analysis of their precision [4], confirmed significantly better accuracy and diagnostic possibilities of the ultrasonic rather than the tearing method of evaluation of adhesive bonds.

\section{Presentation of the problem}

A practical evaluation of the bond condition between the coating and the substrate can be performed with the use of the developed ultrasonic set (Fig. 1), and depends on the diagnostic susceptibility of the whole evaluation system.

As it can be seen in Fig. 1, the evaluation of adhesive bonds can be realized with the use of different kinds of ultrasonic waves under condition of an immediate access to an examined part of machine or by immersion method. However, some examples of industrial evaluation of condition of various adhesive bonds placed in the machine parts already exist, but till now there have not been any models of the ultrasonic diagnosis of adhesive bonds. The necessity of certain formalism of an ultrasonic evaluation in this area appears. Therefore, the purpose of this work is to present some proposals of models of ultrasonic diagnosis of adhesive

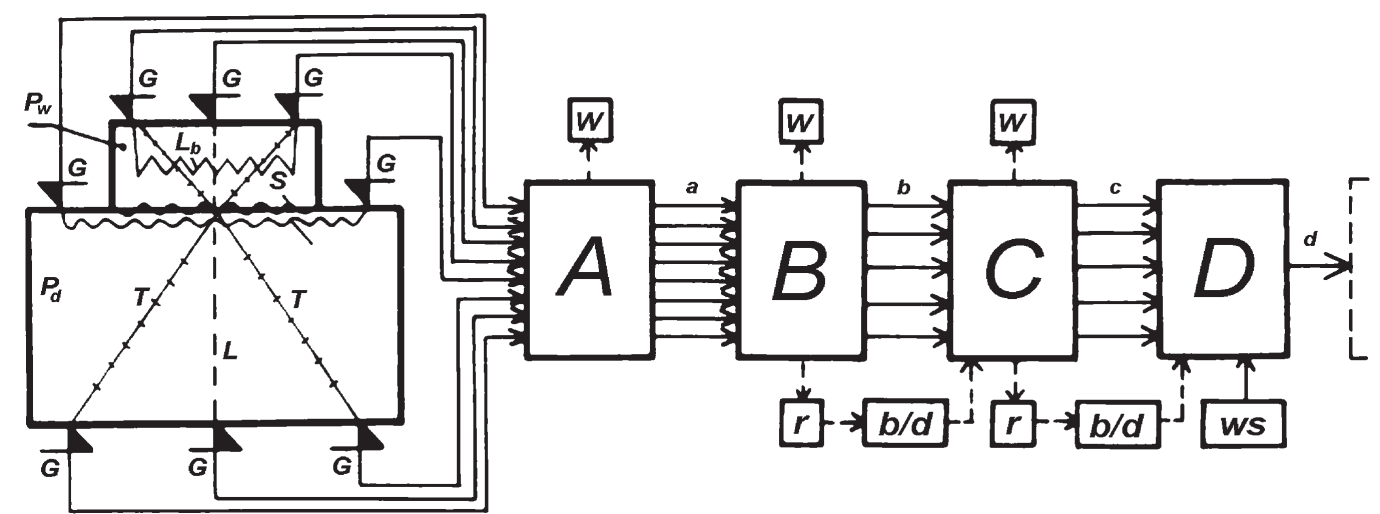

Fig. 1 The measurement set for diagnostics of adhesive bond between two elements: A, B, C, D- respectively: ultrasonic, processing, diagnostic and decision modules, $G$ - ultrasonic probes, $S, T, L$ - respectively: surface, transverse and longitudinal waves

\footnotetext{
* Marian Josko

Poznan University of Technology, Institute of Working Machines and Motor Vehicles, Piotrowo 3, $60-965$ Poznan, Poland, tel. +48 061 6652247, fax. +48 061 6652736, E-mail: Marian.Josko@put.poznan.pl
} 
bonds as a contribution to modelling the diagnosis of these bonds using the ultrasonic method.

\section{The proposal of a control diagnostic model}

This proposal can be generated and presented with aid of some collections of input and output quantities as shown in Fig. 2.

\begin{tabular}{|c|c|c|c|}
\hline INPUT & & & OUTPUT \\
\hline $\begin{array}{l}\text { - Combination of materials } \\
\text { - A set of technological } \\
\text { parameters }\end{array}$ & $Y$ & 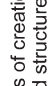 & $\begin{array}{l}\text { - A set of features \& quality } \\
\text { measures of bond }\end{array}$ \\
\hline $\begin{array}{l}\text { - Interference of } \\
\text { production surroundings }\end{array}$ & Z & 这 & $\begin{array}{l}\text { - A set of ultrasonic } \\
\text { symptoms }\end{array}$ \\
\hline
\end{tabular}

Fig. 2 Two collections of input and output quantities connected with forming and evaluation of quality of adhesive bonds during their production

These collections of quantities give us a possibility of the proposal for the diagnostic control model, related to quality control during production of adhesive bonds (Fig. 3). The models presented bellow in this paper can be numbered to a category of socalled black box models.

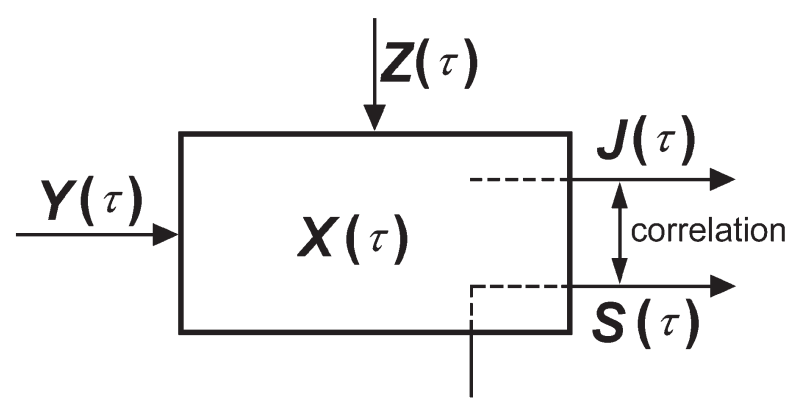

Fig. 3 Graphical presentation of the quality diagnostic model by technical control during production of adhesive bonds (X - a set of structure parameters of adhesive bonds, the other quantities shown here are explained in Fig. 2 and formulas 1,2)

Using an overall form of symptom diagnosis equation [5], the mathematical model of the control diagnostic can be presented as the following dependencies:

$$
\begin{aligned}
& J(\tau)=\Phi[X(\tau), Y(\tau)]+Z(\tau), \\
& S(\tau)=\Phi[X(\tau), Y(\tau)]+Z(\tau),
\end{aligned}
$$

where: $J(\tau)$ - features of bond quality, $\Phi$ - operator of signal transformation (functional of transition), $X(\tau)$ - a set of structural parameters, $Y(\tau)$ - an input set of materials and technological parameters, $Z(\tau)$ - interferences of technological surrounding, $S(\tau)$ - ultrasonic parameters (symptoms), $\tau$ - time of production during which dispersion of quality can occur (dynamics of technological dispersion)

The input set of materials and technological parameters $Y(\tau)$ of the model (Fig. 3) is a collection of parameters of a coat spreading and can be presented in the following form:

$$
Y(\tau)=\left\{T_{1}(\tau), T_{2}(\tau), \ldots, T_{l}(\tau)\right\} .
$$

Parameters $T_{1}(\tau), \ldots, T_{l}(\tau)$, involve a combination of parameters of coating and substrate as a set of technological parameters of coat spreading, ex. parameters and sort of substrate preparation, temperature, distance, angle of tool inclination, etc.

The set of structure parameters $X(\tau)$ consists of all the elements connected with the boundary of adhesive joint, both quality (continuity, defectiveness) and quantity values (forces of adhesion and cohesion, stress and hardness gradient, size and orientation of filler grains). It can be expressed by the following parameters:

$$
X(\tau)=\left\{X_{1}(\tau), X_{2}(\tau), \ldots X_{m}(\tau)\right\}
$$

An evaluation of bonds condition between the coating and substrate by hitherto methods is rather impossible without destruction of examined bonds.

Interferences of technological surroundings $Z(\tau)$ determine a set of such external features as:

$$
Z(\tau)=\left\{Z_{1}(\tau), Z_{2}(\tau), \ldots X_{k}(\tau)\right\}
$$

This set includes such environmental impulses as atmospheric (temperature, humanity), pollutions (dust powders, greasiness), mechanical, physical and chemical effect (aggression), and results of technical and organizational mistakes and all random events.

A collection $J(\tau)$ as a set of quality of adhesive bond contains such elements as follows:

$$
J(\tau)=\left\{J_{1}(\tau), J_{2}(\tau), \ldots J_{n}(\tau)\right\}
$$

This collection comprises such measured features of adhesive bond as the peel, shear and fatigue strengthens, durability, thickness, porosity and permeability of coating. Here are also non-measured characteristics of coatings as its homogeneity, smoothness and an overall appearance. A determination of measured features of quality of the adhesive bonds between coatings and substrate is connected - as rule - with destruction of the bonds and as well as with insight into area of the adhesive bond. The role of generalized measure of quality of adhesive bonds plays mechanical adhesion strength determined in different ways, predominantly by peel strength.

The set of ultrasonic parameters $S(\tau)$ can be described as follows: 


$$
S(\tau)=\left\{S_{1}(\tau), S_{2}(\tau), \ldots S_{p}(\tau)\right\}
$$

The set $\mathrm{S}(()$, described by dependency (7), comprises certain parameters of ultrasonic stimulators, inserted in boarding structures of adhesive bonds in the form of some ultrasonic waves. These waves excite the examined structure and propagate across or along the adhesive boundary. They are received by an ultrasonic probe and can carry information about the condition of such structure as the adhesive bond.

For the purpose of solving the problem of evaluation of the adhesive bond condition, i.e. to determine values of the features of condition of adhesive bond $X(\tau)$ and the characteristics of quality $J(\tau)$ on the basis of values of ultrasonic diagnostic signals $S(\tau)$, the following conditions $[5,6]$ must be satisfied:

$$
\begin{aligned}
& \mathrm{m} \leq \mathrm{n}, \\
& \mathrm{n} \leq \mathrm{p},
\end{aligned}
$$

where: $m$ - a number of independent parameters of bond condition, $n$ - a number of parameters of bond quality, $p$ - a number of ultrasonic symptoms.

During the succeeding control diagnosis, all the components of collection of technological parameters should be maintained on the constant level. Also, the influence of surrounding disturbances is maintained on the constant level or is reduced, or even is minimised. Therefore, assuming - according to [7] - the constancy of two sets of the below-mentioned quantities, namely:

$$
Y(\tau) \cap Z(\tau)=\text { const },
$$

we can obtain a simplified mathematical model of control diagnosis, reducing dependencies $(1,2)$ to the following equations:

$$
\begin{aligned}
& J(\tau)=f[X(\tau)], \\
& S(\tau)=f[X(\tau)] .
\end{aligned}
$$

It means that the output sets parameters $J(\tau)$ and $S(\tau)$ (Figs. 2 and 3 ) depend on the parameters of structure of adhesive bonds $X(\tau)$ and adequate regression equations can be determined by experimental investigations. These investigations enable to obtain the expected relationships between some structural and ultrasonic parameters.

If we assume that the mechanical strength of adhesive bond $p$ is a representative parameter for the quality of adhesive bond, and modulus of the reflection coefficient of ultrasonic wave $|r|$ provides satisfactory information for the evaluation of a condition of adhesive bond, we can present equations (11 and 12) in a more detailed way:

$$
\begin{aligned}
& p(\tau)=f[X(\tau)], \\
& |r|(\tau)=f[X(\tau)] .
\end{aligned}
$$

Then, we are searching relationships between $p(\tau)$ and $|r|(\tau)$.

\section{The proposal of an operational diagnostic model}

This proposal can be generated and presented with aid of some collections of input and output quantities as shown in Fig. 4.

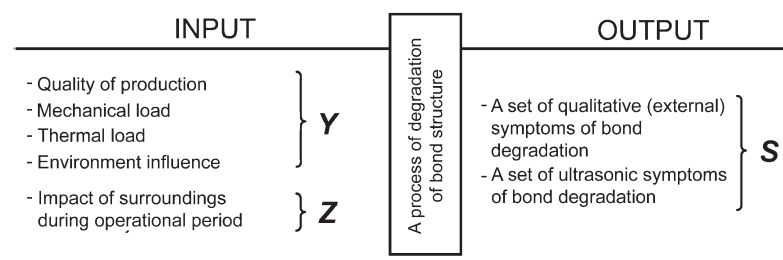

Fig. 4 Two collections of input and output quantities connected with degradation and condition evaluation of adhesive bonds during operational period of their existence

These collections of quantities give us a possibility of the proposal for the operational diagnostic model, related to diagnostic procedure during maintenance of the bonds (Fig. 5).

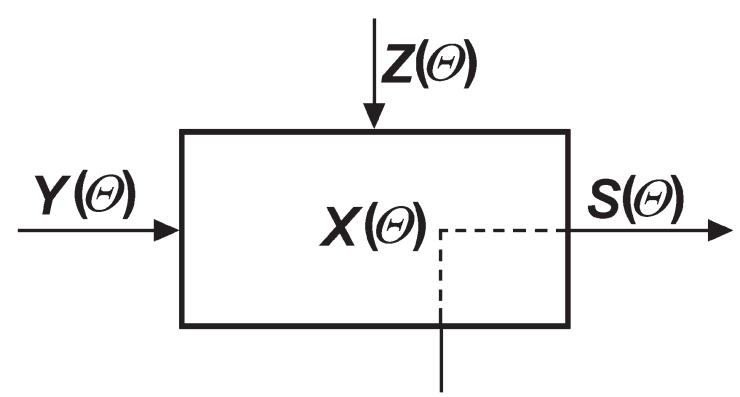

Fig. 5 Graphical presentation of the operational diagnostic model by condition evaluation during maintenance of adhesive bonds ( $X$ - a set of condition parameters of adhesive bonds, the other quantities shown here are explained in Fig. 4 and formula 15)

The model of the operational diagnostic connected with a process of degradation of the adhesive bonds can be presented graphically in Fig 5 and described mathematically with the aid of the following equation [3]:

$$
S(\Theta)=\Phi[X(\Theta), Y(\Theta)]+Z(\Theta),
$$

where: $S(\Theta)$ - ultrasonic symptoms, $\Phi$ - operator of signal transformation (functional of transition), $X(\Theta)$ - a set of features of bond condition, $Y(\Theta)$ - a set of input parameters (quality of production, mechanical, thermal and environmental impact during operational time - Fig. 4), $Z(\Theta)$ - interferences during operational period, $\Theta$ - operational time (period of maintenance of adhesive bond). 
The input collection $Y(\Theta)$ of the operational diagnostic (Fig. 5 ) determines a set of parameters of forces during an operational period, which can be described as follows:

$$
Y(\Theta)=\left\{W_{1}(\Theta), W_{2}(\Theta) \ldots W_{l}(\Theta)\right\} .
$$

Parameters $W_{1}(\Theta) \ldots W_{l}(\Theta)$ are, first of all, dynamics parameters as mechanical forces and moments loaded. Here, thermal loading, environmental influence and deformations caused by mistakes of assembling can be numbered.

The set of features of bond condition $X(\Theta)$ can be expressed by the following formula:

$$
X(\Theta)=\left\{X_{1}(\Theta), X_{2}(\Theta) \ldots X_{m}(\Theta)\right\}
$$

The set $X(\Theta)$ comprises the whole quality and quantity relations between both connected materials, namely the coating and the substrate. These relations are determined by mechanical, adhesive, cohesive and chemical connections on the boundary and in the adjoined areas of the bonds. The main measure of these connections is the strength of bond for the particular kind of destructive load.

All interferences during the operational period $Z(\Theta)$ include the following collection of features:

$$
Z(\Theta)=\left\{Z_{1}(\Theta), Z_{2}(\Theta) \ldots Z_{k}(\Theta)\right\}
$$

These interferences, which belong to $Z(\Theta)$, are dynamics, thermal and environmental impulses from so called near-by (direct) surroundings of machine parts including adhesive bonds. They have a form of random vibrations, aggressive influence of surroundings, overloads or failure ex. seizing.

The collection of ultrasonic symptoms of bond condition $S(\Theta)$ can be described in the following way:

$$
S(\Theta)=\left\{S_{1}(\Theta), S_{2}(\Theta) \ldots S_{n}(\Theta)\right\} .
$$

As in the previous example, i.e. the control diagnostic model, this collection consists of a set of parameters of ultrasonic stimulators in the different form of ultrasonic waves. Selected ultrasonic parameters can be symptoms of bond condition, because they are sensitive to the degraded changes of the adhesive bonds during the operational period considered.

With the aim of solving the diagnostic problem of condition evaluation of the adhesive bonds by ultrasonic method during the operational period, i.e. determination of values of some condition features $X(\Theta)$ on the basis of values of ultrasonic parameters $S(\Theta)$, the following condition must be satisfied $[5,6]$ :

$$
m \leq n,
$$

where: $m$ - a number of independent parameters of bonds condition, $n$ - a number of ultrasonic parameters (symptoms).
In order to simplify the operational diagnostic model (Fig. 5) and its mathematical notation (15) during the normal operational process, we can assume minimization or even stabilization of all disturbances from the surroundings. Such approach is motivated in references $[5,7]$. It can be also assumed that for the succeeding times $\Theta$, when the operational diagnosis will be carried out, the elements of the set $Y(\Theta)$ should be constant in respect of the number and value. Constant values of the input set for succeeding evaluations of bonds state is an essential condition of repeatability of ultrasonic diagnosis of the adhesive bonds analysed. Therefore, permitting a possibility of stability of two sets of input quantities, namely:

$$
Y(\Theta) \cap Z(\Theta)=\text { const },
$$

a simplified mathematical recording of the graphical operational model shown in Fig. 5 and written by (15), can be presented finally as the following equation:

$$
S(\Theta)=f[X(\Theta)] .
$$

It means that output parameters, i.e. visual and ultrasonic symptoms $S(\Theta)$ are dependent only on parameters of condition of the adhesive bonds $X(\Theta)$. It is necessary to receive some dependencies between parameters of condition of the adhesive bonds and parameters of ultrasonic waves using both visual and ultrasonic techniques for monitoring of the bonds.

For this purpose should be planed a kind of active diagnostic experiments aimed at receiving some collections of the run of ultrasonic parameters during an operational time, dependent on condition of the adhesive bonds.

\section{The proposals of procedures of ultrasonic diagnoses of adhesive bonds}

The first proposal, in the case of using longitudinal ultrasonic wave for evaluation of bonds condition during control diagnosis, the procedure of qualification of the adhesive bonds to appropriate state is presented by schematic diagram in Fig. 6 .

The second proposition is grounded on realization of some ultrasonic measurements of adhesive bonds during their operational (maintenance) period by ultrasonic surface wave, according to the procedure shown in Fig. 7.

Both proposed procedures allow us to qualify the examined adhesive bonds using commercial ultrasonic probes for longitudinal or surface waves and main ultrasonic parameters, namely a modulus of reflection coefficient $|\mathrm{r}|$ and a gain of ultrasonic impulse W. The selection of diagnostic model and the procedure of ultrasonic diagnosis of adhesive bonds depend on the period of life-time of machine parts comprising the bonds analysed.

\section{Conclusions}

The diagnostic approach to adhesive bonds evaluation allows us to formalize the problem of evaluation of coating adherence, 


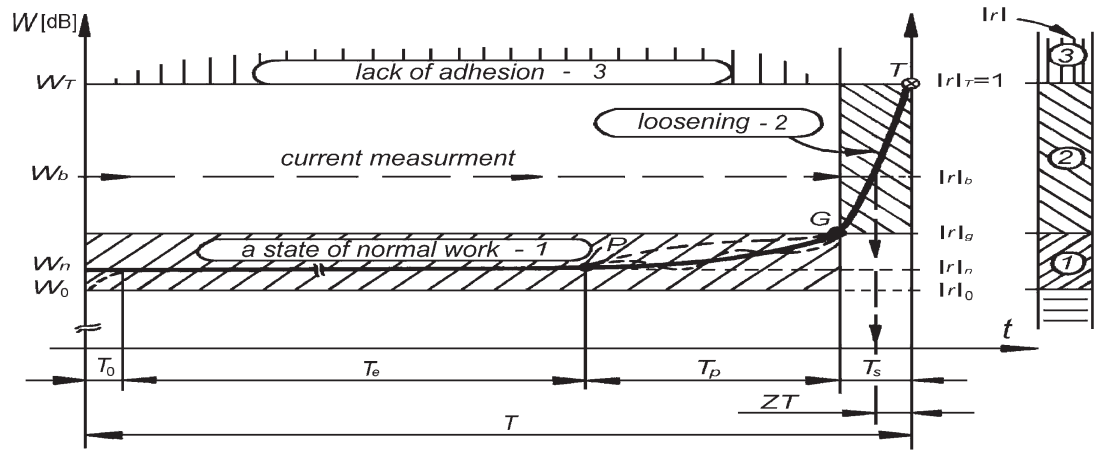

Fig. 6 The schematic diagram of the procedure of realization of bonds condition by ultrasonic longitudinal wave during control diagnosis of adhesive bonds by ultrasonic measurements: numerals 1, 2, 3 mean respectively: good, weak and bad adhesive bonds; $W$ - ultrasonic parameter, $t$ - time of a bond existence, $T$ - durability of a bond, $|r|$ - modulus of reflection coefficient

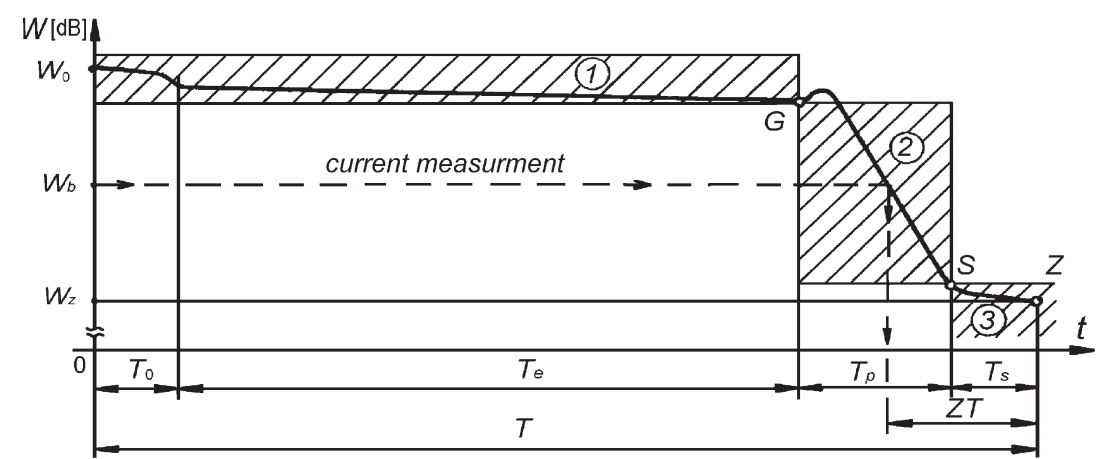

Fig. 7 The scheme of the proposed procedure of operational diagnosis of adhesive bonds by ultrasonic measurements: numerals 1, 2, 3 mean respectively: good, weak and bad adhesive bonds; $W$ - ultrasonic parameter, $t$ - time of existence of a bond, $T$ - durability of a bond

reducing that problem to the assessment of the condition of adhesive bonds between coatings and substrate at the various stages of their existence.

Two complementary models proposed, both in graphical and mathematical forms, reflect two periods of existence of the adhesive bonds, namely period of their production and period of their operational (maintenance) time.
Some equations obtained in this work motivate experimental investigations with the aim to obtaining relationships between parameters of the structure of adhesive bonds and selected ultrasonic parameters which can be understood as symptoms of condition of the adhesive bonds.

\section{References}

[1] JOSKO M.: An Ultrasonic Assessment of Adhesive Bonds between Coating and Substrate, Proceedings of the Conference Transcom 97, University of Zilina, 1997, Vol. 3, pp. 261-264.

[2] JOSKO M.: Correlation between an Ultrasonic Signal and Adhesive Strength (in Polish), Proceedings of 28th Polish Conference of Nondestructive Testing, Warsaw, 1999, pp. 103-106.

[3] JOSKO M.: Methodological Aspects of Evaluation of Regenerative Coatings Adherence Using an Ultrasonic Method (in Polish), PP Poznan, 2002.

[4] JOSKO M.: Investigation of the Degradation of Joints by Ultrasonic Method, Proceedings of the Conference Transcom-99, University of Zilina, 1999, Section 6, pp. 47-50.

[5] ZOLTOWSKI B.: A Basis of Technical Diagnostics (in Polish), ATR, Bydgoszcz, 1996.

[6] CEMPEL C.: Data Reduction in Machine Diagnosis (in Polish), Zagadnienia Eksploatacji Maszyn (Polish Journal), 1980 Vol. 15, No. 4, pp. 571-585.

[7] TOMASZEWSKI F.: Determination of Technical Condition of Complex Technical Object (in Polish), PP, Poznan, 1998. 